Revista Calidad en la Educación Superior

Programa de Autoevaluación Académica

Universidad Estatal a Distancia, Costa Rica

ISSN 1659-4703

revistacalidad@uned.ac.cr

\title{
LA ACREDITACIÓN DE POSGRADOS EN CIENCIA POLÍTICA EN ARGENTINA
}

\author{
ACREDITATION OF POLITICAL SCIENCE GRADUATE COURSES IN ARGENTINA
}

\author{
Sebastián M. Rinaldi ${ }^{1}$ \\ sebastianmrinaldi@hotmail.com \\ Universidad Nacional de La Matanza y \\ Universidad de Palermo, Argentina
}

Volumen 6, Número 2

Noviembre 2015

Pp. $45-63$

Recibido: 2 de junio, 2015

Aprobado: 22 de setiembre, 2015

\footnotetext{
1Sebastián M. Rinaldi, especialista en Didáctica y Currículum (UNLZ, Argentina). Licenciado en Ciencia Política (UNLaM, Argentina). Diplomado Semi-presencial en Investigación en Infancia y Juventud para miembros de las instituciones latinoamericanas pertenecientes a la Red Childwatch (CINDE-Universidad de Manizales, Colombia). Profesor de la carrera de Ciencia Política en la Universidad Nacional de La Matanza y la Universidad de Palermo. Miembro del equipo técnico de acreditación de posgrados de la Comisión Nacional de Evaluación y Acreditación Universitaria (CONEAU). Correo electrónico: sebastianmrinaldi@hotmail.com
} 


\section{Resumen}

En el presente artículo se realiza una revisión de los procesos de acreditación de las carreras de posgrado en Ciencia Política ante la Comisión Nacional de Evaluación y Acreditación Universitaria (CONEAU), un organismo descentralizado que actúa en jurisdicción del Ministerio de Educación de la Nación Argentina.

Se trata de un estudio descriptivo en el cual se caracterizan los principales aspectos de las evaluaciones y se identifican los resultados de las mismas, destacándose las fortalezas y las debilidades detectadas en las presentaciones realizadas por cada una de las instituciones universitarias, a fin de contribuir al conocimiento de la situación actual de los posgrados de la disciplina y profundizar la línea de estudio sobre el currículum de esta carrera que se viene desarrollando durante los últimos años.

Palabras clave: Evaluación; Acreditación; Posgrado; Ciencia Política; Currículum.

\section{Abstract}

In this paper, a review of the processes of accreditation of graduate programs in Political Science at the National Commission of University Assessment and Accreditation (CONEAU), decentralized agency acting under the Ministry of Education of Argentina, takes place.

In this descriptive study the main aspects of the assessments are characterized while its results are identified, highlighting the strengths and weaknesses detected in the presentations made by each university, to contribute to the knowledge of the current situation of postgraduate studies of the discipline and to go in depth with the line of research in the curriculum of this field which is being developed in recent years.

Keywords: Assessment; Accreditation; Graduate Programs; Political Science; Curriculum.

\section{Introducción}

La actual agenda de la educación universitaria se fue constituyendo a partir de la incidencia de distintos factores políticos, sociales y económicos que condujeron a revisar el vínculo entre el Estado, las universidades y la sociedad civil durante las tres últimas décadas (CONEAU, 2011). En este contexto, la calidad y su evaluación fueron ganando terreno en el ámbito de la educación superior a nivel mundial, en un proceso en el que se fue comprendiendo gradualmente la necesidad de formar cada vez más y mejores egresados para actuar conforme a las demandas profesionales del mercado laboral y de la sociedad 
misma (Aiello, 2011). Con esta premisa, surgieron diversas agencias privadas y públicas con el objetivo de asegurar y mejorar la calidad de la formación superior.

En Argentina, a partir de la aprobación de la Ley 24.521 de Educación Superior (LES) en 1995, se introdujeron la evaluación y el aseguramiento de la calidad como nuevos ejes de la política universitaria. De este modo, por medio de la LES, se creó la Comisión Nacional de Evaluación y Acreditación Universitaria (CONEAU), un organismo descentralizado que actúa en jurisdicción del Ministerio de Educación de la Nación, y que comenzó a funcionar en agosto de 1996.

En la actualidad, esta Comisión concentra una serie de funciones entre las que se destacan la evaluación externa de universidades, la evaluación de proyectos institucionales, la acreditación de carreras de grado cuyos títulos corresponden a profesiones reguladas por el Estado, y cuyo ejercicio pudiera comprometer el interés público (art. 43 de la LES), y la acreditación de todas las carreras de posgrado del país. Sobre esta última función se centra el presente artículo.

El posgrado en Argentina resulta ser una de las realidades menos explorada en los estudios sobre educación superior. En parte, esto se debe al número reducido de estudiantes que tiene el nivel de posgrado frente a la gran masa de estudiantes de grado (concentrando por su dimensión mucho más la atención de las investigaciones), pero también a la expansión heterogénea, explosiva y poco planificada que han experimentado las ofertas de posgrado en el país durante las últimas décadas (De la Fare \& Lenz, 2012), transformándose en un fenómeno difícil de abordar cuantitativa y metodológicamente.

En este caso, y en función de otros trabajos que se han venido realizando con antelación sobre la carrera de ciencia política en el ámbito universitario (Rinaldi, 2014; 2015a; 2015b), se decidió describir algunos aspectos sobresalientes de los procesos de acreditación de todos los posgrados de la disciplina en el país. Para ello, se tomaron la totalidad de las presentaciones de las instituciones universitarias ante la CONEAU, en las que esta se 
expidió otorgando o no la acreditación, para efectos de identificar las principales fortalezas y debilidades que se evidenciaron.

El propósito de este trabajo es continuar con la línea de trabajo que se viene desarrollando sobre el currículum universitario de ciencia política, abordando por primera vez el mayor nivel de formación académica, con la finalidad de contribuir al conocimiento de la situación actual de los posgrados de la disciplina.

\section{Educación de posgrado}

El posgrado es el máximo nivel de formación académica, cuyas funciones principales son la provisión de recursos humanos de alta calidad en sus diferentes niveles (especialización, maestría y doctorado) y la generación de docentes para la educación superior e investigadores (Morles, 1997; Ruíz-Nápoles, 1997).

A diferencia de los estudiantes de grado, quienes acceden a este nivel educativo, deben tener necesariamente una formación profesional previa, lo que implicaría que cada sujeto encuentre motivaciones bien distintas de aquellos que inician un ciclo de licenciatura. Así, se reconoce que quienes optan por realizar un posgrado, lo hacen por razones de actualización (buscando una renovación sistemática de conocimientos y habilidades profesionales con los avances y el desarrollo científico), de complementación (logrando la adquisición de nuevos conocimientos y habilidades profesionales no recibidos en estudios precedentes o adquiridos sin la profundización requerida) y/o de profundización (intentando obtener un nivel superior en los conocimientos científico-técnicos y habilidades profesionales en el campo específico de una profesión o de un área concreta de la ciencia y la técnica) (Organización Panamericana de la Salud, 1992).

El desarrollo científico y tecnológico, los cambios en los sistemas productivos y las nuevas formas de generar conocimiento reclaman hoy nuevos profesionales con capacidad de adaptarse a un mundo fluctuante y dar soluciones a los problemas más relevantes. Y es en el 
nivel de posgrado donde se presentan las condiciones necesarias para poder problematizar la realidad y producir respuestas nuevas (Saavedra, 2006).

En el caso argentino, el sistema de posgrado ha venido creciendo fuertemente desde la década de 1990, observándose en él una oferta desordenada y, en muchos casos, superpuesta en un proceso de claro desajuste con el grado. Esta situación fue generada porque desde un principio la regulación de la oferta de posgrados quedó librada a las políticas desarrolladas por las universidades, con frecuencia más relacionadas a una cuestión de mercado (presentándose este nivel como una gran fuente adicional de ingresos para las casas de altos estudios), incluida la propia demanda de títulos por los integrantes del sistema académico, careciendo generalmente de una articulación con las instituciones que conforman el sistema de ciencia y tecnología (Barsky \& Dávila, 2009).

Los posgrados acreditados que se dictan en la actualidad en el país son 2688, entre los que se contabilizan 411 doctorados, 906 maestrías y 1351 especializaciones (CONEAU, 2015). Si bien, el número sigue siendo elevado y todo indica que a futuro seguiría creciendo, en los últimos años los procesos de evaluación han colaborado a evitar una proliferación desordenada y sin sentido. No obstante, continúan evidenciándose problemáticas que han sido frecuentes desde los orígenes de este sistema: las bajas tasas de egreso (que en algunos casos son nulas), acompañadas por la falta de políticas institucionales orientadas a evitar la deserción y el desgranamiento, la insuficiencia de becas para alumnos (con posgrados que resultan ser muy costosos, incluso los de las universidades de gestión estatal), la ausencia de retribuciones para los docentes por la dirección de las tesis, la no incorporación de estudiantes a los equipos de investigación, entre otros (De la Fare \& Lenz, 2010).

En la actualidad, el proceso de expansión de las carreras de posgrado se relaciona con la creciente demanda de personal calificado por parte del Estado y del sector productivo. Respondiendo a la complejidad del mundo actual, este nivel educativo resulta ser un lugar que potencia el desarrollo de nuevas disciplinas, de la interdisciplinariedad, de la 
especialización y diversificación del saber (Dávila, 2009). Se conforma así un sistema diverso que debe convivir con las continuas adaptaciones a la sociedad de la información y la comunicación, el surgimiento de nuevos campos de estudio en los cuales se debaten espacios de trabajo, la evolución de las disciplinas más tradicionales, las nuevas demandas sociales y de mercado, la integración internacional (que impulsa los procesos de migración de docentes y alumnos) y la presión que suponen los procesos de evaluación y acreditación (López-Segrera, 2006).

Frente a este complejo panorama, se plantea la necesidad de abandonar el tradicional concepto de universidad, asociado a una instancia de reproducción y certificación de conocimientos, para reconocerla como un escenario en el que se brinden nuevas oportunidades educativas en el nivel universitario a lo largo de la vida de las personas y con la mejor calidad posible.

\section{La calidad en la educación superior}

El debate sobre la calidad en el campo de la educación superior se instaló fuertemente en la década de 1980, como resultado del impacto de la globalización del capitalismo informacional en los sistemas educativos. Desde entonces, la educación comenzó pensarse como un servicio sujeto a las presiones del mercado y, por lo tanto, como una mercancía más que requiere competir en precio y calidad con otros servicios (Andión-Gamboa, 2007).

Ante esta realidad, se comenzó a hablar del "Estado Evaluador" para referirse al análisis de fenómenos que acontecían en la educación superior europea como una forma de coordinación y regulación de los sistemas de educación superior, reforzándose la relación Estado-Universidad. Esto se daba en un contexto en el que se dotaba de mayor autonomía a las instituciones para el control de sus procesos, pero a la vez se instalaban sistemas de evaluación para juzgar el uso de dicha autonomía atendiendo a los resultados obtenidos.

En la región latinoamericana, la inclusión de estos mecanismos se produjo en un contexto político, económico y financiero sumamente complejo, "frecuentemente asociado a políticas 
de restructuración del aparato estatal y de racionalización y selectividad del gasto público" (Betancur, 1998). Así, la evaluación se presentaba inicialmente como una política que no solo limitaba las posibilidades de autonomía institucional, sino que además se consideraba una herramienta de desfinanciamiento de la educación superior.

Sin embargo, hoy en día, la visión respecto de la existencia de mecanismos de evaluación ha cambiado notablemente. Las agencias han ido ganando su espacio y reconocimiento en el entramado estatal y del sistema educativo, como aquellas que permiten mejorar y elevar continuamente el nivel y la calidad de los procesos educativos, que a la vez generan una reflexión constante en cada una de las universidades para la revisión de la propia estructura de funcionamiento y de los programas académicos, permitiendo el acopio de experiencias y destrezas para otras instancias de evaluación. Las instituciones, en líneas generales, han entendido la importancia de someterse a estos procesos y el impacto en el desarrollo de estas que puede ocasionar: acceso a programas de becas y financiamiento, una mayor cantidad de inscritos en carreras de posgrado (que supone mayores ingresos), o bien, mayor prestigio, son algunas de las consecuencias posibles.

Este cambio, respecto de la concepción de la evaluación, obedece en gran parte a la clarificación de los criterios que se han ido implementando para medir la calidad de las ofertas educativas: el establecimiento de estándares mínimos por parte de la comunidad educativa para todas las carreras de posgrado (distinguiendo según el nivel, ya sea especialización, maestría o doctorado) que reglan los procesos de evaluación; el número de egresados, la calidad de sus trabajos finales y su inserción profesional; el número de ingresantes y el volumen de becas otorgadas, reconociendo los grados de democratización en el acceso a la educación superior de posgrado (Rinaldi, Tonon, Aragón \& Suárez, 2013); el nivel de actualización y formación del cuerpo académico junto con su dedicación a la investigación (Vain, 2000); las facilidades que cada institución pone a disposición del alumnado para vehiculizar su cursado; el grado de pertinencia curricular, vinculándose las propuestas del espacio educativo con las características y las necesidades del medio 
ambiente en el cual se inserta (Méndez-Fregoso, 2005); entre otros, son algunos componentes claves en esta política.

\section{El proceso de acreditación en Argentina}

La Resolución Ministerial № 160/11 (Ministerio de Educación de la Nación, 2011) establece los estándares a aplicar en los procesos de acreditación de carreras de posgrado y fija las etapas del procedimiento de la acreditación.

A este proceso se deben presentar todas aquellas carreras nuevas que tengan la intención de dictarse como así también aquellas que se encuentran en funcionamiento y su período de acreditación esté por expirar o no hayan sido acreditadas anteriormente.

En el período de las convocatorias que realiza la CONEAU, cada unidad académica debe presentar la documentación en la que consta la información básica de la carrera (e. g. plan de estudios, autoridades, cuerpo docente, convenios específicos, trabajos finales, infraestructura, planes de mejora) en formato físico complementado con información digital.

Pasado un tiempo, cada carrera es asignada a un técnico de la CONEAU, el cual está a cargo de establecer contacto con los pares evaluadores cuyos perfiles reúnen las características necesarias para evaluar determinadas carreras. Dichos pares, son elegidos del registro de expertos que la institución ha ido conformando a lo largo de estos años con los candidatos que las universidades sugieren de su plantel de docentes e investigadores. Al día de hoy, el registro está integrado por poco más de 12.000 expertos de diversas profesionales. Para asegurar la transparencia del proceso, ningún experto puede evaluar a su institución de pertenencia ni a aquellas que se encuentran en la misma región geográfica donde este desempeña su actividad profesional.

Más tarde, a lo largo de toda una semana, se realizan los comités, donde se reúnen expertos de un mismo campo con las carreras que les fueron asignadas y proceden a realizar la 
evaluación con la asistencia del técnico de CONEAU. En dicha instancia, se realizan los informes de evaluación donde los expertos sugieren o no la acreditación de cada propuesta de formación de posgrado. Este encuentro permite aunar criterios entre los profesionales de un área disciplinar con la intención de lograr consistencia en los criterios que se utilizarán para observar y juzgar las presentaciones de las instituciones. Asimismo, durante esos días, se realizan entrevistas a las autoridades de los posgrados con la finalidad de esclarecer aquellas cuestiones que generaran dudas, tanto en los técnicos como en los evaluadores. En algún caso, y de considerarse necesario, los miembros del comité y el personal de CONEAU pueden desplazarse hasta las instalaciones donde se dictarán los posgrados a fin de poder tener más insumos para lograr un proceso de evaluación satisfactorio.

Luego de que el comité se expide, los resultados pueden ser los siguientes:

a) Que la carrera acredite por un plazo de 3 años, si son carreras nuevas o aún no tienen egresados, o 6 años, para las que se encuentran en funcionamiento y, además, tienen egresados. A su vez se les puede otorgar categoría si es que la solicitaron en la presentación de la documentación (A si se considera excelente, B si es muy buena y C si es buena).

b) Que la carrera vaya a vista (lo que implica que la institución observe el informe de los pares para reconocer aquellas cosas que debe corregir para poder ser acreditada).

Si la carrera acreditó, se realiza la resolución correspondiente que es aprobada por los miembros de la Comisión (un directorio de 12 personas responsables de la institución que son elegidos en representación de diferentes esferas del arco político y educativo nacional, encabezados por un Presidente que renueva periódicamente su cargo). De lo contrario, la institución debe responder a la vista que se le dio del expediente y nuevamente un par debe (con la asistencia de un técnico) realizar el informe de vista en el cual se puede recomendar o no su acreditación, observando si se subsanaron las cuestiones que se le advirtieron en el informe de evaluación. 
Nuevamente, si los cambios son suficientes, se realiza la resolución correspondiente que otorga la acreditación a la carrera. De no considerarse adecuadas las modificaciones informadas en la respuesta a la vista de la institución, se realiza una resolución que establece la no acreditación. En este último caso, cada universidad/unidad académica tiene una instancia de recurso de reconsideración, ante la cual la Comisión puede ratificar o rectificar su fallo.

A lo largo de todo este proceso, se evalúa el grado en el que cada carrera se adecua a la normativa ministerial como así también la pertinencia de cada propuesta con las exigencias que debe reunir, a juicio de CONEAU y de los pares evaluadores, un posgrado en cada una de las áreas del conocimiento: producción científica, promoción de mayores grados de experticia, acceso para los estudiantes al estado del arte disciplinar, factibilidad de implementación de la propuesta de formación, trabajos finales y/o tesis que supongan un aporte al desarrollo de la ciencia, la existencia de masa crítica en las instituciones, la concreción de los mecanismos de seguimiento del desempeño de docentes, alumnos y egresados, pertinencia curricular, grado de profesionalización del cuerpo académico y docente, entre otros, son los principales aspectos qué se evalúan en el marco de las acreditaciones. Todos estos criterios dan cuenta de que la evaluación se trata de una instancia en la que se intenta asegurar y mejorar la calidad de las carreras e instituciones universitarias que operan en el sistema universitario argentino.

\section{Las presentaciones de los posgrados en Ciencia Política ante CONEAU}

Para el presente estudio se consideraron solamente aquellas carreras en la cuales se pudo distinguir claramente que el objeto de estudio era la política misma. Por eso, se tuvo en cuenta que la denominación y los contenidos remitieran estrictamente a la ciencia política, no considerándose ofertas que implicaran subdisciplinas o áreas específicas como la comunicación política, las políticas públicas, la administración pública, la gestión, entre otras, de las cuales existen numerosos posgrados en el país y se pretenden abordar en estudios posteriores. 
Teniendo en cuenta lo dicho, se puede afirmar que desde la creación de la CONEAU se registraron 41 presentaciones de carreras de posgrado en ciencia política, entre especializaciones (4), maestrías (18) y doctorados (19), de 16 universidades distintas (9 de gestión privada y 7 de estatal).

Cuadro 1. Cantidad de carreras presentadas por institución

\begin{tabular}{|c|c|}
\hline Institución & $\begin{array}{c}\text { Cantidad de } \\
\text { presentaciones }\end{array}$ \\
\hline Universidad Católica Argentina & 5 \\
\hline Universidad Nacional de General San Martín & 4 \\
\hline Universidad Torcuato Di Tella & 4 \\
\hline Facultad Latinoamericana de Ciencias Sociales & 4 \\
\hline Instituto Universitario ESEADE & 4 \\
\hline Universidad Nacional de La Plata & 3 \\
\hline Universidad Nacional de Córdoba & 3 \\
\hline Universidad del Salvador & 3 \\
\hline Universidad Nacional de Rosario & 2 \\
\hline Universidad de Belgrano & 2 \\
\hline Universidad Nacional de La Rioja & 2 \\
\hline Universidad de San Pablo-Tucumán & 1 \\
\hline Universidad Católica de Santa Fe & 1 \\
\hline Universidad Nacional del Nordeste & 1 \\
\hline Universidad Maimónides & 1 \\
\hline Universidad Nacional de Río Cuarto & 1 \\
\hline
\end{tabular}

Fuente: Elaboración propia.

En cuanto al tipo de presentación, se observó una gran diversidad: proyectos de carreras, carreras en funcionamiento que se presentaron por primera vez y carreras que incluso ya van por su segunda y tercera presentación ante CONEAU.

Del total de posgrados que se evaluaron, 24 resultaron acreditados, 2 recibieron el reconocimiento oficial provisorio (por ser proyectos) y los restantes no acreditaron o se encuentran actualmente en alguna de las instancias de evaluación. 
Respecto de las categorías que recibieron los posgrados acreditados, se advierte que los resultados evidencian cierta paridad. Mientras que las categorías $A$ y $B$ fueron otorgadas a 4 carreras cada una, la $C$ fue otorgada a 5. Las restantes no solicitaron categoría o bien se trató de proyectos, los cuales no son considerados para la categorización.

Por otra parte, si se tiene en cuenta el plazo por el cual las carreras fueron acreditadas, se aprecia que la mitad ha recibido 3 años y el resto 6 . En algunos casos, el hecho de que se les otorgara solo 3 años se debió a su condición de carreras nuevas, sin embargo, también se pudo determinar que hay carreras que al momento de la evaluación no habían logrado aún obtener egresados, condición necesaria para la obtención de la máxima cantidad de tiempo posible de acreditación.

Otro dato que merece ser destacado es la gran concentración territorial de las ofertas. Más de la mitad de las ofertas que se presentaron (27), pertenecen a universidades de la región metropolitana. Esto se debe, al igual que sucede con otras ofertas de posgrado, a las mayores posibilidades de demanda de realización de carreras del nivel que se encuentran en los grandes centros urbanos, donde además confluyen gran parte de las casas de altos estudios que funcionan en la actualidad en el país.

Por último, se debe destacar que no todas las carreras evaluadas remiten solamente a la ciencia política en su denominación y contenido. En algunos casos (7), se identificaron titulaciones en interdisciplina con otros campos, distinguiéndose especializaciones y maestrías asociadas a los campos de las ciencias jurídicas, la sociología y la economía.

\section{Fortalezas y debilidades de las carreras}

El proceso de evaluación por el que atravesaron cada una de las carreras a las que se hace referencia en este estudio, permitió detectar las principales características de las propuestas académicas, resaltándose aquellos aspectos positivos y a mejorar por parte de las instituciones. 
En primer lugar, uno de los datos significativos que se puede extraer de la lectura de las presentaciones, es la calidad de los cuerpos académicos. En los informes de evaluación rara vez se ha objetado el nivel de los docentes y de los directivos. En el caso de los doctorados, casi la totalidad del cuerpo académico tenía el título de doctor y antecedentes en investigación científica. Respecto de los directores, se reconoce su participación en organismos de ciencia y tecnología (CONICET o Sistema de Incentivos), una gran trayectoria docente en grado y posgrado, experiencia en gestión de la educación superior, y antecedentes más que suficientes en dirección de tesis y producción académica. Asimismo, los docentes dan cuenta de su trayectoria en dirección de tesis doctorales, contribuyendo a las condiciones para facilitar la realización de las mismas, y la posterior graduación.

De esta manera, a medida que se desciende en el nivel académico, el plantel docente y el cuerpo académico suele acreditar menor cantidad de antecedentes como investigadores, en producciones académico-científicas y direcciones de trabajos finales o tesis. Por otra parte, los planteles de especializaciones y maestrías suelen, además, tener menor cantidad de personal. Esto último puede obedecer a que como gran parte de los doctorados suelen tener tramos personalizados, la oferta de materias puede ser superior a la cantidad de materias que podrían encontrarse en los planes de estudios de maestrías profesionales (diferenciadas de las académicas por sus objetivos prácticos por sobre la producción científica) o especializaciones, y de allí el mayor número de docentes.

Uno de los núcleos claves en el análisis de la información que presentan las instituciones es aquel referido a la investigación. Si bien los doctorados y las maestrías académicas tienen investigaciones suficientes, las especializaciones prácticamente no registran actividades de este tipo vinculadas a la carrera. Esto se debe, en parte, a que la Res. Ministerial № 160/11 no exige a este nivel académico el desarrollo de actividades de investigación asociadas a la formación, principalmente por tratarse de una formación vinculada estrictamente al desarrollo de habilidades prácticas para el ejercicio profesional. Sin embargo, debe remarcarse que 
aquellas carreras que si las tienen pueden recibir el reconocimiento en el proceso de evaluación, por ejemplo, en la consideración de la categoría a otorgar.

Asimismo, se reconoce en gran parte de los casos abordados que, independientemente del nivel académico, las carreras registran una baja participación de los alumnos en los proyectos de investigación. Por un lado, esto obedece a la falta de políticas institucionales destinadas a incluirlos dentro de los programas o proyectos acreditados, aunque también, en el caso particular de los doctorados, existen varios alumnos que realizan su formación como becarios bajo el financiamiento de la Comisión Nacional de Investigaciones Científicas y Técnicas (CONICET) u otro programa de formación de investigadores, por lo cual tienen una dedicación exclusiva a esa actividad en ámbitos distintos al de la propia institución donde realizan la carrera en cuestión.

En este contexto, se advierte que en las resoluciones de la CONEAU se suele recomendar incentivar las actividades de investigación en el marco de la carrera y la participación de los alumnos en las mismas, junto con el desarrollo de procesos de transferencia (e. g. jornadas, publicaciones). Pero para ello, resulta fundamental que se aumente la dedicación de los docentes a la carrera contando con un cuerpo de profesionales que puedan dirigir proyectos vinculados con las temáticas de los posgrados, algo que se remarca en diferentes juicios de evaluación.

Por otra parte, las bajas tasas de graduación suelen ser una preocupación en los tres grados académicos, razón por la cual, en casi todos los informes de evaluación se recomienda que se tomen medidas tendientes a incrementar el número de egresados. Para ello, se sugieren una serie de medidas que podrían ser útiles para el abordaje de la problemática: una evaluación integral de los mecanismos de evaluación de desempeño de los alumnos, dedicación efectiva de los docentes estables, la inclusión de un seminario de tesis en los casos en los que no se contempla, instrumentos de apoyo y promoción a los alumnos (como las becas y los subsidios que suelen ser escasos), y dedicación efectiva exclusiva de éstos a 
la carrera, pueden contribuir a identificar y resolver dificultades y mejorar el ritmo de graduación.

Un último punto que se quisiera destacar, y que los expertos mencionaron en sus juicios, es que en algunos casos los requisitos de admisión resultaban ser muy amplios (e. g. graduados universitarios), no limitando el ingreso a profesionales provenientes de las ciencias sociales. Sin embargo, en un par de doctorados de la disciplina se observó un alto grado de exigencia, puesto que se requería experiencia previa en otros niveles de posgrado y/o investigación en el área temática, e incluso el manejo de idiomas. Por lo dicho, se puede afirmar que en el área disciplinar no suelen haber acuerdos concretos sobre que rasgos debería tener un profesional que decide acceder a una instancia de formación como las estudiadas, ni de parte de las mismas instituciones ni en los criterios de aquellos que han participado en los procesos de evaluación.

\section{Consideraciones finales}

En el presente artículo, se realizó una primera revisión sobre las diferentes presentaciones que se han realizado de posgrados en ciencia política ante el organismo acreditador nacional.

Las carreras evaluadas en el campo de estudio difieren en características y resultados, aunque se observan algunas regularidades en cuanto a sus aciertos y desaciertos. En comparación a otras ciencias sociales, el porcentaje de carreras que han acreditado ante la CONEAU es bastante elevado, algo que habla de la calidad de los posgrados que se han venido dictando durante las últimas décadas en el país.

Con cuerpos académicos y docentes sumamente capacitados, los posgrados en ciencia política tienen las condiciones para formar estudiantes que pueden convertirse en recursos humanos valiosos para el desarrollo de la disciplina desde su ejercicio profesional. Sin embargo, y a pesar de la larga tradición de investigación que tiene la Argentina en materia de 
política, se reconoce que ese debe ser un aspecto a profundizar en los diferentes espacios de formación académica, destinando esfuerzos a incrementar el número de docentes y estudiantes avocados a este tipo de actividad.

Asimismo, se identifican tasas de graduación muy bajas. Si bien esto fue remarcado en el desarrollo del trabajo como una realidad común a otros campos, cada carrera debe procurar dedicar esfuerzos a resolver este problema en función de la realidad que atraviesa su comunidad de estudiantes. En general, se reconoce que los alumnos de estas ofertas suelen ser empleados vinculados a la administración o la función pública, la participación política partidaria o la gestión institucional, actividades que demandan mucho tiempo y reducen las posibilidades de una terminalidad exitosa de los posgrados. Y es allí, en la identificación del perfil del posgraduante, donde se debe hacer hincapié para mejorar, no solo las tasas de graduación, sino las ofertas de carreras en su totalidad. Programas de becas, mecanismos administrativos de seguimiento de alumnos, tutorías personalizadas, son solo algunas de las posibilidades.

Este trabajo, en definitiva, permitió el abordaje de una realidad inexplorada como son los posgrados en ciencia política en el país y sus procesos de acreditación, abriendo la posibilidad de continuar con el estudio de la temática a partir de la identificación de las principales ofertas y características de los ciclos de formación, en un esfuerzo por reconocer la calidad de los procesos educativos.

\section{Recomendaciones}

Para vehiculizar una mejora constante de las ofertas de posgrado en ciencia política, es imprescindible que las instituciones logren identificar las mayores dificultades sobre las cuales seguir trabajando.

Uno de los principales aspectos a considerar es la revisión constante del plan de estudios a los efectos de mantenerlo actualizado y en relación a los temas centrales que prevalecen en 
otras formaciones de su tipo. Se ha evidenciado con anterioridad, por ejemplo, que la reiteración de temas y autores entre materias del mismo ciclo de grado es algo frecuente (Rinaldi, 2015a), cuestión crucial al momento de pensar la conformación de un proyecto de posgrado. Los ciclos de especializaciones, maestrías y doctorados, a pesar de las diferencias que tienen en cuanto a su complejidad y los objetivos específicos que persiguen, tienen la misión de formar recursos humanos altamente calificados. En este sentido, no resulta lógico que en los posgrados se vean contenidos temáticos que responden a niveles inferiores de formación académica.

Respecto del plan de estudios, una cuestión que suele ser bastante controversial es la que refiere a la estructura de cursado. Frecuentemente se observa como diferentes propuestas organizan la asistencia a clases de manera intensiva en pocos días al mes, y en otros casos generan una frecuencia semanal o quincenal. Independientemente del tipo de estructura por la cual se termina optando, la discusión pasa por ver si se privilegia un sistema que los estudiantes puedan sostener a lo largo de los meses o la adaptabilidad a los horarios de los profesores del programa. Con seguridad, afirmamos que el foco debe estar puesto en generar las condiciones adecuadas para promover en los estudiantes un desarrollo y una terminalidad exitosa de carrera, pensando un sistema que no desaliente la continuidad sin que esto suponga un detrimento en la calidad de la oferta.

Asimismo, resulta sumamente importante para las universidades que se ajusten los criterios para evaluar aspirantes durante el proceso de admisión, en función del bajo nivel de egreso que manifiestan las carreras. El criterio más importante, a nuestro entender, es la factibilidad de realización y aprobación de todas las actividades que implica el posgrado en tiempo y forma, producto de la combinación de factores tales como el tipo de trabajo que tiene el postulante, su situación familiar y la distancia entre su lugar de vivienda/trabajo y el lugar de cursado, su formación previa, su producción profesional y/o académica vinculada a la temática de la carrera, y sus antecedentes en haber iniciado y concluido (o no) anteriormente otro ciclo de formación de posgrado. 
Las bajas tasas de graduación también son producto de deficientes mecanismos de acompañamiento de los posgraduantes, no solo en la confección del documento final escrito, sino a lo largo del proceso de formación. Las formas que tiene una dirección de carrera de supervisar estos aspectos son muy variadas: tutorías, seguimiento del desempeño a través de informes de los docentes, asistencia a las clases, entrevistas periódicas.

\section{Referencias}

Aiello, M. (2011). La evaluación de instituciones de educación superior en Argentina: comparación entre los procesos de evaluación de instituciones universitarias y de institutos de formación docente. IV Congreso Nacional y III Encuentro Internacional de Estudios Comparados en Educación. ¿Hacia dónde va la Educación en la Argentina y en América Latina? Construyendo una nueva agenda. Buenos Aires, 16 y 17 de junio.

Andión, M. (2007). Sobre la calidad en la educación superior: una visión cualitativa. Reencuentro, 50, 83-92.

Barsky, O. \& Dávila, M. (2009). La evaluación de posgrados en la Argentina. Documento de trabajo № 226. Buenos Aires: Universidad de Belgrano.

Betancur, N. (1998). El Estado evaluador como nueva forma de relacionamiento EstadoUniversidades. Revista Uruguaya de Ciencia Política, 10, 117-131.

Comisión Nacional de Evaluación y Acreditación Universitaria (2011). La CONEAU y el sistema universitario argentino: memoria 1996-2011. Ciudad Autónoma de Buenos Aires: CONEAU.

Comisión Nacional de Evaluación y Acreditación Universitaria (2015). Posgrados acreditados de la República Argentina. Ciudad Autónoma de Buenos Aires: CONEAU.

Dávila, M. (2009). Tendencias internacionales de la educación superior. Documento de trabajo № 219. Buenos Aires: Universidad de Belgrano.

De la Fare, M. Lenz, S. (2010). La política de posgrado en la Argentina y la expansión de carreras. VI Jornadas de Sociología de la UNLP. Universidad Nacional de La Plata

De la Fare, M. \& Lenz, S. (2012). El posgrado en el campo universitario. Expansión de carreras y productividad de tesis en la Argentina. Los Polvorines: Universidad de General Sarmiento.

Gobierno de la Nación Argentina (1995). Ley de Educación Superior № 24.521. 
López-Segrera, A. (2006). Escenarios mundiales de educación superior. Análisis global y estudios de casos. Buenos Aires: CLACSO.

Méndez-Fregoso, E. (2005). La pertinencia como requisito para la calidad en Educación Superior. La planeación institucional y el compromiso como condición esencial para el desarrollo del posgrado. Revista Iberoamericana de Educación, 36 (3).

Ministerio de Educación de la Nación (2011). Resolución №160/11.

Morles, V. (1997). La producción intelectual como finalidad esencial del postgrado en América Latina. En Morles, V. (Comp.), Postgrado y desarrollo en América Latina. CEISEA 4 (Pp. 38-45). Caracas: Ediciones del Centro de Estudios e Investigaciones sobre Educación Avanzada (CEISEA), Coordinación Central de Estudios de Postgrado, Universidad Central de Venezuela.

Organización Panamericana de la Salud (1992). Los cambios de la profesión y su influencia sobre la educación médica. Washington (DC).

Rinaldi, S., Tonon, G., Aragón, S. \& Suárez, A. (2013, octubre). Políticas públicas y Calidad de vida universitaria: El caso de la Facultad de Ciencias Sociales de la UNLZ. XI Jornadas IDICSO. Universidad del Salvador.

Rinaldi, S. (2014). Sobre la confección y la defensa de trabajos finales en Ciencia Política. Ficha de Cátedra. Universidad de Palermo.

Rinaldi, S. (2015a). El currículum de Ciencia Política en las universidades nacionales argentinas: Un estudio de casos colectivo. Tesis de Maestría en Currículum. Universidad Nacional de Lomas de Zamora, Buenos Aires, Argentina.

Rinaldi, S. (2015b). Las prácticas pre-profesionales en las licenciaturas en Ciencia Política. Revista Iberoamericana de Educación Superior, Instituto de Investigaciones sobre la Universidad y la Educación, de la Universidad Nacional Autónoma de México. ISSN 2007-2872 (A la espera de aceptación).

Ruíz-Nápoles, P. (1997). El papel de la universidad pública en relación con el posgrado y el desarrollo económico. En Morles, V. (Comp.), Postgrado y desarrollo en América Latina. CEISEA 4, 21-29. Caracas: Ediciones del Centro de Estudios e Investigaciones sobre Educación Avanzada (CEISEA), Coordinación Central de Estudios de Postgrado, Universidad Central de Venezuela.

Saavedra, M. (2006). Posgrados y demandas laborales: La evaluación de los profesionales. Revista Iberoamericana de Educación, 38 (4),1-14.

Vain, P. (2000). La evaluación de la docencia universitaria: un problema complejo. Serie Estudios № 4. Ciudad Autónoma de Buenos Aires: CONEAU. 